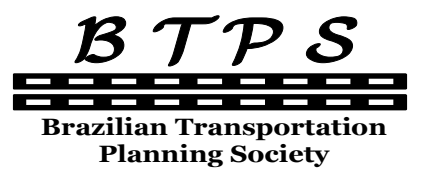

Journal of Transport Literature

Vol. 8, n. 4, pp. 7-36, Oct. 2014

Research Directory
JTL|RELIT

www.journal-of-transport-literature.org ISSN 2238-1031

\title{
Principais indicadores que afetam a percepção da qualidade de serviço em áreas críticas do embarque de passageiros aeroportuários
}

[Key indicators that affect the perception of service quality in critical airport areas of passenger boarding]

\author{
Michelle Carvalho Galvão da Silva Pinto Bandeira*, Giovanna Miceli Ronzani Borille, \\ Marina Ciarlini Duarte Maia, Rodrigo Flório Moser \\ Aeronautics Institute of Technology - Brazil, Aeronautics Institute of Technology - Brazil, \\ Aeronautics Institute of Technology - Brazil, National Civil Aviation Agency - Brazil
}

Submitted 10 Sep 2012; received in revised form 14 Feb 2013; accepted 18 Oct 2013

\section{Resumo}

O presente trabalho selecionou três importantes aeroportos brasileiros para pesquisas de campo, observação e entrevista com passageiros nas áreas de check-in e inspeção de segurança. 0 objetivo do trabalho foi obter a importância de indicadores, qualitativos ou quantitativos, que afetam a percepção da qualidade de serviço no Aeroporto Internacional de São Paulo (SBGR), Aeroporto de São Paulo/Congonhas (SBSP) e Aeroporto Internacional de Brasília (SBBR). A metodologia adotada foi: (i) seleção dos indicadores, (ii) método de coleta, (iii) dimensionamento da amostra e, (iv) método de regressão linear. Os resultados finais revelaram que os modelos obtidos indicaram que há uma relação forte entre a percepção, ou seja, a visão que o passageiro tem sobre o componente e/ou serviço oferecido no terminal de passageiros e o tempo decorrido, seja este de espera na fila ou de atendimento. Os resultados mostraram ainda, que cada aeroporto apresentou variáveis independentes diferentes atuando fortemente sobre as mesmas variáveis dependentes dos modelos analisadas. Adicionalmente, alguns indicadores avaliados neste trabalho não foram relatados na literatura, trazendo uma contribuição importante para os resultados, além de subsídios para a melhoria destes serviços.

Palavras-Chave: aeroportos brasileiros, indicadores de qualidade, check-in, inspeção de segurança, regressão linear.

\begin{abstract}
This paper selected three major Brazilian airports for field research, observation and interviews with passengers in the areas of check-in and security screening. The objective was to obtain the importance of indicators, qualitative or quantitative, that affect the perception of service quality at the International Airport of São Paulo (SBGR), Airport of São Paulo/Congonhas (SBSP) and Brasilia International Airport (SBBR). The procedures used for the research were:: (i) selection of indicators, (ii) collection method, (iii) scaling the sample and, (iv) regression method. The final results show that the models obtained indicated that there is a strong relationship between perception, that is, the view that the passenger is about the component and /or service offered in the passenger terminal and the time - wait time in queue or time of attendance The results also showed that each airport presented different independent variables, acting in the same heavily dependent variables of the models considered. Additionally, some indicators evaluated in this study were not reported in the literature, obtaining an important contribution to the results, and subsidies to improve these services.
\end{abstract}

Key words: Brazilian airports, quality indicators, check-in, security screening, linear regression.

*Email: michelle_galvao@yahoo.com.br.

\section{Recommended Citation}

Bandeira, M., Ronzani, G. M., Ciarlini, M. and Moser, R. F. (2014) Principais indicadores que afetam a percepção da qualidade de serviço em áreas críticas do embarque de passageiros aeroportuários. Journal of Transport Literature, vol. 8, n. 4, pp. 7-36.

- JTL/RELIT is a fully electronic, peer-reviewed, open access, international journal focused on emerging transport markets and published by BPTS - Brazilian Transport Planning Society. Website www.journal-of-transport-literature.org. ISSN 2238-1031. 


\section{Introdução}

O estudo apresenta uma análise sobre a percepção de passageiros aeroportuários em duas das principais áreas de embarque - check-in e inspeção de segurança. Utilizou-se, para tanto, indicadores qualitativos e quantitativos, coletados durante a pesquisa de campo com passageiros que estavam realizando algum dos procedimentos citados. O método de regressão linear foi aplicado e os resultados dos modelos indicaram forte relação entre a percepção e o tempo decorrido dos passageiros nestas áreas dos aeroportos analisados.

Observa-se que, o processamento de passageiros nas operações de embarque e desembarque no terminal podem tornar-se gargalos por diversos fatores, como por exemplo, o congestionamento no lado ar, a demora no atendimento do check-in, as instalações de processamento de bagagens indisponíveis, o sistema inoperante entre outros. Isso pode ocasionar desde pequenos atrasos e/ou concentração de pessoas em níveis toleráveis até a completa insatisfação dos usuários. Nesse caso, pode-se dizer que a capacidade do terminal está comprometida, mesmo que por um dado período de tempo.

Dessa forma, a avaliação da capacidade de processamento do terminal está diretamente relacionada ao nível de serviço que o aeroporto oferece ao passageiro. Logo, obter a relação de importância que os indicadores têm sobre os componentes aeroportuários mais críticos é extremamente necessário para a melhoria global do aeroporto.

Tem-se observado, ainda, que o crescimento da demanda pelo transporte aéreo tem exigido cada vez mais um aumento da capacidade do sistema aeroportuário. A construção de novos aeroportos ou a ampliação de instalações em um determinado aeroporto, visando a atender esse crescimento, certamente acarretaria um aumento dessa capacidade (Carvalho, 2006).

No entanto, esta não é uma diretriz fácil de ser executada, pois questões econômicas, políticas e/ou ambientais são dimensões que devem ser consideradas. A implementação de projetos dessa magnitude envolve a interferência de entidades governamentais, bem como de diversos setores da sociedade, além do tempo demandado para esta implementação. Desse modo, com o crescimento do transporte aéreo e, consequentemente, do movimento nos terminais 
aeroportuários, tornam-se necessários estudos visando à otimização dos procedimentos operacionais e um melhor aproveitamento das áreas disponíveis (Medeiros, 2004).

A atual pesquisa selecionou três importantes terminais de passageiros (TPS) de aeroportos brasileiros, sendo o Aeroporto Internacional de São Paulo/Guarulhos - Governador André Franco Montoro (SBGR), Aeroporto de São Paulo/Congonhas (SBSP) e Aeroporto Internacional de Brasília/Presidente Juscelino Kubitschek (SBBR). Estes três aeroportos juntos representam, aproximadamente, 39\% do movimento de passageiros que utilizaram, em 2008, o modal aéreo no Brasil (ANAC, 2009). Como citado no Relatório de Movimento Operacional da ANAC (ANAC, 2009), estes são considerados, para o caso brasileiro, como aeroportos de "extra grande porte", pois desde 2004 processaram mais de 8 milhões de passageiros por ano. Ao analisar os dados deste mesmo relatório observa-se que, desde 2005, estes aeroportos estão entre os três principais no ranking de movimento de passageiros por ano no que diz respeito ao tráfego doméstico e internacional contabilizado no país.

O objetivo do trabalho foi obter o grau de importância de indicadores qualitativos e quantitativos que afetam a percepção da qualidade de serviços das áreas de check-in e inspeção de segurança do processamento de embarque dos aeroportos selecionados na pesquisa. Desta modo, foram encontrados resultados acerca das percepções e perspectivas de diferentes tipos de passageiros, como os que realizaram check-in em balcões de atendimento ou em totens de autoatendimento, por exemplo.

Este trabalho está assim estruturado: a Seção 1 trata de uma revisão bibliográfica sobre métodos de análises qualitativas e/ou quantitativas. A Seção 2 apresenta os detalhes da pesquisa de campo e a aplicação da regressão linear. A Seção 3 aborda os resultados encontrados; por fim, apresentamos as conclusões finais. 


\section{Nível de serviço em terminais aeroportuários}

Vários estudos sobre o nível de serviço em terminais de passageiros foram realizados por muitas agências internacionais, como a Federal Aviation Administration (FAA, 1988), Airports Council Internacional (ACI, 2000), International Civil Airports Association (ICAA, 1979), Transport Canada (1979) e Transportation Research Board (TRB, 1987) mas tais estudos e métodos propostos foram desenvolvidos sem consultar a opinião dos usuários. Por outro lado, um número considerável de pesquisadores tem desenvolvido vários métodos para avaliar e priorizar os componentes aeroportuários e seus indicadores sob a ótica do usuário, os principais foram comentados a seguir.

Mumayiz e Ashford (1986) apresentaram um método chamado de conceito perceptionresponse (P-R), através de gráficos construídos a partir da opinião dos passageiros sobre o nível de serviço de alguns aeroportos da Inglaterra. Apesar de haver consulta ao passageiro, as respostas dadas pelos usuários não foram capazes de analisar vários componentes ou indicadores do TPS ao mesmo tempo, pois este método só permite a avaliação de único atributo.

Omer e Khan (1988) buscaram capturar a importância relativa dos componentes aeroportuários através da opinião dos usuários sobre o nível de serviço percebido, mas empregaram o conceito de utilidade para desenvolver um relacionamento entre características dos componentes (tempo de espera, espaço disponível) e as opiniões dos passageiros sobre o nível de serviço oferecido.

Estes trabalhos, pioneiros, foram importantes na incorporação da pesquisa de opinião com passageiros durante os procedimentos de embarque. No entanto, os métodos aplicados não foram suficientes para obter a importância relativa de cada componente ou atributo avaliado. Diante do exposto, o presente estudo incorporou a pesquisa de opinião dos passageiros que participaram dos procedimentos de check-in ou inspeção de segurança como parte da metodologia.

Segundo a FAA (1988), o componente check-in é uma área crucial para as companhias aéreas e trata-se de um dos objetivos iniciais para a maioria dos passageiros que chegam ao aeroporto. Dentro desse escopo, recomenda-se que os balcões de check-in estejam bem 
posicionados de forma a oferecer ao passageiro um acesso imediato, com curtas distâncias de percurso do meio fio até o balcão e com fácil visibilidade (Edwards, 1998).

Wells (1996) observou que alguns fatores podem influenciar na operação e na capacidade das atividades de check-in, tais como: o número de unidades e a disposição dos balcões, os procedimentos de operação das companhias aéreas, as características e o perfil dos passageiros, o espaço e o tipo de configuração, o tipo de voo, os acordos com a administração aeroportuária as práticas de gerenciamento.

Yeh e Kuo (2003) utilizaram lógica fuzzy para avaliar o nível de serviço em 14 aeroportos internacionais da Ásia-Pacífico, onde cada critério agregava vários atributos semelhantes, tais como: conforto, tempo de processamento, conveniência, cortesia do pessoal, visibilidade da informação e segurança, entre outros.

Correia (2005) desenvolveu uma proposta de medida global de nível de serviço através da utilização de fatores objetivos (tempo de processamento, espaço disponível, distância de percurso, etc.) e uso de técnicas de regressão linear. Bandeira et al (2007) utilizou o método AHP (Analytical Hierarchy Process) para calcular a importância relativa entre os indicadores dos principais componentes do Aeroporto Internacional de São Paulo/Guarulhos. Ronzani e Correia (2007) avaliaram o indicador "orientação do passageiro" em terminais de 11 aeroportos brasileiros, por meio de pesquisa com passageiros e técnicas multivariadas.

A partir das pesquisas citadas, observou-se que um número maior de atributos em pesquisas de campo com passageiros foram analisados, onde atributos semelhantes podem estar atrelados a um determinado componente aeroportuário. Desse modo, a atual pesquisa avalia diversos atributos por tipo componente, agregando à análise aspectos qualitativos e quantitativos.

Segundo de Neufville e Odoni (2003), a evolução tecnológica pode tornar os tradicionais balcões de check-in obsoletos. De fato, torna-se importante salientar a existência de outras formas de procedimentos de check-in, como os totens de autoatendimento e as possibilidades de check-in via internet (WEB check-in). Com a propagação destas novas opções, pode ocorrer a redução da necessidade de grandes halls, dos balcões de check-in e do tempo de atendimento. Além disso, o check-in de autoatendimento vem se tornando comum, por meio 
do esforço de companhias aéreas em reduzir custos operacionais e melhorar o desempenho do fluxo de embarque de passageiros no TPS.

O presente estudo considerou, dentro do terminal aeroportuário do País, totens de autoatendimento durante as pesquisas de campo. Esta abordagem agrega relevância aos resultados, uma vez que a implantação dos totens de autoatendimento no País tem sua maior utilização por parte dos passageiros apenas nos últimos 5 anos. Uma referência pioneira na literatura pode ser encontrada em Magri Junior (2008). Tal estudo utilizou o uso de técnicas de regressão linear para avaliar os impactos da aplicação de check-in de autoatendimento no Aeroporto de São Paulo/Congonhas. No entanto, o autor não comparou os resultados encontrados com o check-in tradicional, feito por balcões de atendimento no mesmo período.

Ainda em relação ao check-in tradicional, é importante ressaltar, o conceito de sistema CUTE (Common Use Terminal Equipament). Conforme a IATA (2004), o CUTE é um termo genérico da indústria da aviação, cujo intuito é permitir o compartilhamento de balcões de diferentes companhias aéreas, quando a programação de voos permitir. O princípio deste conceito é a possibilidade de empresas aéreas dividirem entre si determinadas instalações do TPS, como as aéreas de check-in e os balcões localizados nos portões de embarque. Segundo Magri Junior (2008), este conceito de uso comum de sistemas, permite que agentes de empresas aéreas utilizem o sistema de outras empresas para a realização de uma operação, no entanto, para que isso ocorra o software operacional deve ser o mesmo.

Outro componente avaliado neste estudo é a área de inspeção de segurança, de extrema importância para garantir um alto nível de proteção para o aeroporto. Visto que, a inspeção de passageiros e de bagagens de mão é necessária antes do embarque na aeronave. $\mathrm{O}$ aeroporto deve garantir a segurança contra atos ilícitos, por meio de equipamentos como dispositivos eletrônicos, máquinas e pórticos de Raio-X, no momento da vistoria de passageiros e bagagens de mão (Horonjeff e Mckelvey, 1993).

Alguns fatores podem influenciar no nível de serviço e na capacidade das áreas de inspeção de segurança, tais como: o número de canais ou posições, o espaço disponível, o pessoal (qualificação e treinamento), o tipo de equipamento, políticas, práticas e/ou procedimentos, as características e o perfil dos passageiros, o layout da edificação e o modelo de circulação e a programação do voo. 
Dada a relevância dos componentes operacionais de embarque, nos quais são desempenhadas importantes funções de operação do TPS, esta pesquisa concentra-se nas áreas de check-in e de inspeção de segurança. Sobre este último, destaca-se ainda, que o enfoque foi dado no processamento de passageiros e de bagagens de mão, procedimento que ocorre através das máquinas e pórticos de Raio-X.

A próxima seção apresenta a descrição da metodologia utilizada na obtenção dos resultados. Levou-se em consideração o levantamento de indicadores da literatura, as características físicas dos aeroportos selecionados e as necessidades atuais das áreas de check-in e inspeção de segurança dos terminais aeroportuários.

\section{Metodologia da pesquisa}

Para obter os resultados deste trabalho, foram selecionados indicadores - qualitativos e quantitativos - que afetam a percepção da qualidade de serviço. Os indicadores foram selecionados a partir do levantamento da literatura e reuniões com especialistas do setor aeroportuário. A pesquisa, levou em consideração aspectos tecnológicos - check-in em autoatendimento, check-in pela internet ou pelo celular. Dessa forma, indicadores como habilidade do funcionário em totens de autoatendimento, tempo de fila de despacho de bagagens, entre outros foram observados durante a pesquisa em campo.

As áreas selecionadas para a coleta de dados no TPS foram as áreas check-in e inspeção de segurança, obtendo-se o tempo gasto pelo passageiros e a opinião do serviço em cada um dos procedimentos citados no Aeroporto Internacional de São Paulo/Guarulhos - Governador André Franco Montoro (SBGR), Aeroporto de São Paulo/Congonhas (SBSP) e Aeroporto Internacional de Brasília/Presidente Juscelino Kubitschek (SBBR). O dimensionamento da amostra foi determinado pela estimativa da proporção populacional de cada um dos aeroportos, onde foi aceito um erro real de até 6\% por aeroporto. Por último, os resultados finais foram obtidos por meio da técnica de Regressão Linear, apresentados a seguir.

Os procedimentos usados para a pesquisa destacam os seguintes passos: i. seleção dos indicadores; ii. método de coleta; iii. dimensionamento da amostra; e iv. método de regressão linear. 


\subsection{Seleção dos indicadores}

Os indicadores selecionados para o projeto foram estabelecidos, a priori, por meio do levantamento de literatura; e, a posteriori, definidos em reuniões com consultores e especialistas do setor. Segundo a revisão de literatura e reuniões técnicas definiram-se os indicadores de estudo para os componentes check-in e inspeção de segurança.

Parte da contribuição deste trabalho encontra-se nesta fase de seleção de indicadores, visto que alguns indicadores são inéditos, relatados nesta pesquisa pela primeira vez, contribuindo, assim, como um referencial para estudos posteriores. As Tabelas 1 e 2 foram obtidas por meio de um levantamento dos principais indicadores utilizados na literatura, desde 1979. As células hachuradas mostram os indicadores quantitativos e qualitativos, respectivamente, utilizados para o check-in tradicional; e a Tabela 3, os indicadores utilizados na literatura para a inspeção de segurança.

Observa-se na literatura para a avaliação do nível de serviço em check-in tradicional, que os indicadores: "forma de organização da fila", "espaço para fila", "sinalização do balcão", "localização do balcão", "quantidade de funcionários em atendimento", "formas de priorização no atendimento", "clareza sobre as informações do voo" e "aparência da área de check-in", não citados ou avaliados em outros estudos. Isto foi verificado também para a avaliação do nível de serviço em inspeção de segurança, onde os indicadores "forma de organização" e "confiança nos procedimentos de vistoria" também são inéditos.

Ao todo foram selecionados 11 indicadores qualitativos e 2 indicadores quantitativos para o check-in tradicional; 08 indicadores qualitativos e 04 indicadores quantitativos para o check-in autoatendimento e 06 indicadores qualitativos e 02 indicadores quantitativos para a inspeção de segurança, como podem ser visto na Tabela 4. As Tabelas 5, 6 e 7 definem os indicadores relacionados para as áreas check-in tradicional, check-in de autoatendimento e inspeção de segurança, respectivamente ${ }^{1}$.

\footnotetext{
${ }^{1}$ Todas as tabelas apresentadas neste trabalho são de autoria dos autores.
} 
Tabela 1 - Principais indicadores quantitativos para avaliação do check-in tradicional

\begin{tabular}{|c|c|c|c|c|c|c|c|c|c|c|}
\hline & INDICADORES & $\begin{array}{l}\text { Quantidade } \\
\text { de balcões }\end{array}$ & $\begin{array}{c}\text { Largura } \\
\text { do balcão }\end{array}$ & $\begin{array}{l}\text { Profundidade } \\
\text { do balcão }\end{array}$ & $\begin{array}{c}\text { Área de } \\
\text { ocupação }\end{array}$ & $\begin{array}{c}\text { Distância entre } \\
\text { passageiros na } \\
\text { fila } \\
\end{array}$ & $\begin{array}{l}\text { Comprimento } \\
\text { de fila }\end{array}$ & $\begin{array}{c}\text { Tempo de } \\
\text { espera na } \\
\text { fila } \\
\end{array}$ & $\begin{array}{l}\text { Tempo de } \\
\text { atendimento }\end{array}$ & $\begin{array}{l}\text { Espaço de } \\
\text { circulação }\end{array}$ \\
\hline \multirow{11}{*}{ 空 } & ICAA (1979) & & & & & & & & & \\
\hline & Alves (1981) & & & & & & & & & \\
\hline & TRB (1987) & & & & & & & & & \\
\hline & FAA (1988) & & & & & & & & & \\
\hline & INFRAERO (1988) & & & & & & & & & \\
\hline & Widmer e Silva (1989) & & & & & & & & & \\
\hline & IATA (1995) & & & & & & & & & \\
\hline & Seneviratne e Martel (1995) & & & & & & & & & \\
\hline & ACI (2000) & & & & & & & & & \\
\hline & IATA (2004) & & & & & & & & & \\
\hline & Medeiros (2004) & & & & & & & & & \\
\hline
\end{tabular}


Tabela 2 - Principais indicadores qualitativos para avaliação do check-in tradicional

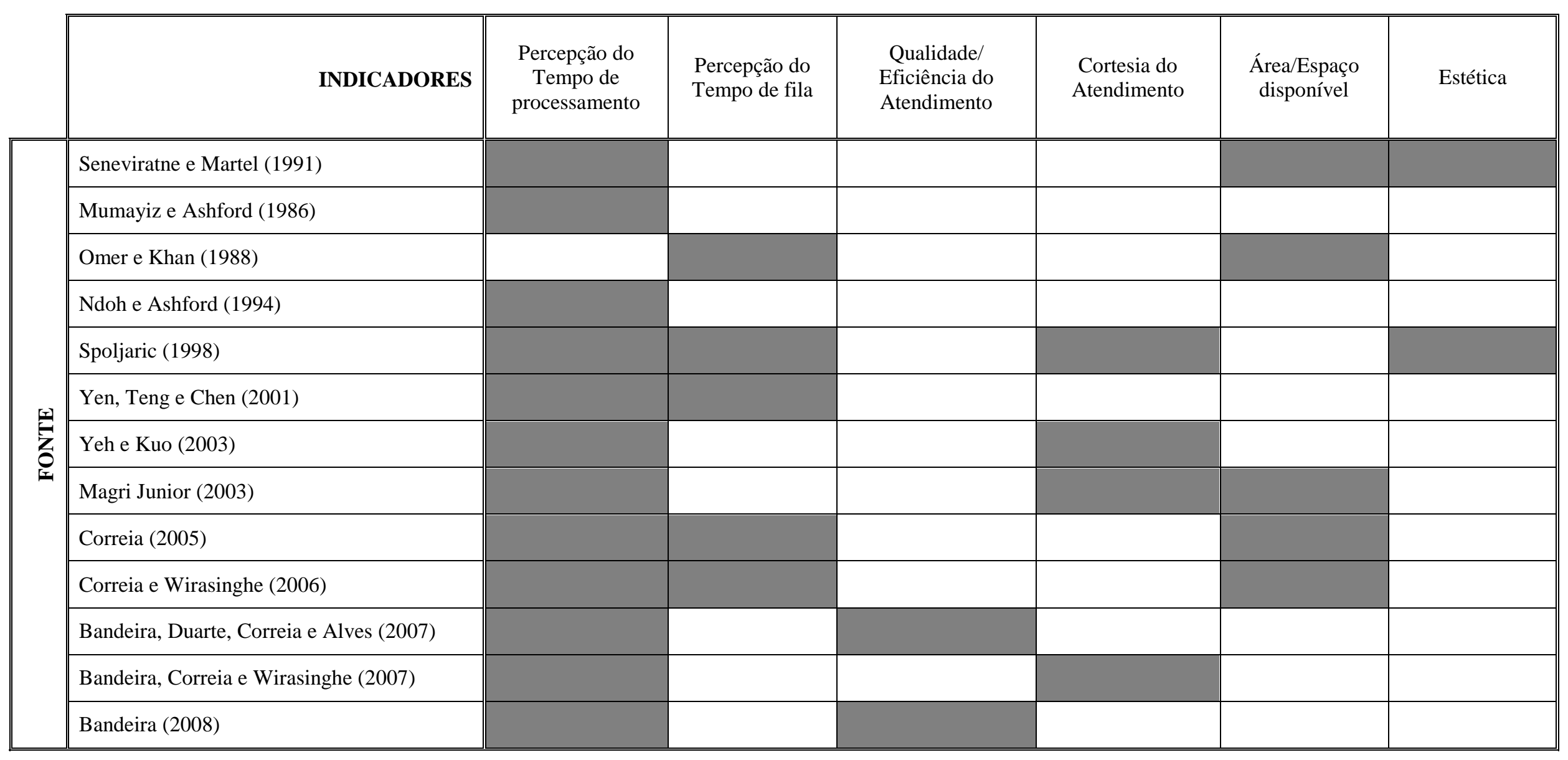


Tabela 3 - Principais indicadores para avaliação da área de segurança em terminais aeroportuários

\begin{tabular}{|c|c|c|c|c|c|c|c|c|c|c|c|}
\hline INDICADORES & $\begin{array}{l}\text { Área/ } \\
\text { Espaço }\end{array}$ & $\begin{array}{l}\mathrm{N}^{\circ} \text { de } \\
\text { Posiçõ̃es }\end{array}$ & $\begin{array}{l}\mathrm{N}^{\circ} \text { de } \\
\text { Pessoal }\end{array}$ & $\begin{array}{l}\mathrm{N}^{\circ} \text { de } \\
\text { pessoas } \\
\text { na fila }\end{array}$ & $\begin{array}{l}\text { Qualificação } \\
\text { do Pessoal }\end{array}$ & $\begin{array}{c}\text { Cortesia } \\
\text { do } \\
\text { Pessoal }\end{array}$ & $\begin{array}{l}\text { Eficiência } \\
\text { do Pessoal }\end{array}$ & $\begin{array}{l}\text { Qualidade } \\
\text { do Serviço }\end{array}$ & $\begin{array}{l}\text { Qualidade dos } \\
\text { Equipamentos }\end{array}$ & $\begin{array}{c}\text { Tempo de } \\
\text { Processamento }\end{array}$ & $\begin{array}{c}\text { Percepção } \\
\text { da } \\
\text { Segurança }\end{array}$ \\
\hline Alves (1981) & & & & & & & & & & & \\
\hline Mumayiz e Ashford (1986) & & & & & & & & & & & \\
\hline TRB (1987) & & & & & & & & & & & \\
\hline FAA (1988) & & & & & & & & & & & \\
\hline INFRAERO (1988) & & & & & & & & & & & \\
\hline Martel e Seneviratne (1991) & & & & & & & & & & & \\
\hline IATA (1995) & & & & & & & & & & & \\
\hline ACI (2000) & & & & & & & & & & & \\
\hline Lee e Kim (2003) & & & & & & & & & & & \\
\hline Magri Junior (2003) & & & & & & & & & & & \\
\hline Yen e Kuo (2003) & & & & & & & & & & & \\
\hline Medeiros (2004) & & & & & & & & & & & \\
\hline Yoo e Choi (2006) & & & & & & & & & & & \\
\hline Correia et al (2007) & & & & & & & & & & & \\
\hline De Barros e Tomber (2007) & & & & & & & & & & & \\
\hline
\end{tabular}


Tabela 4 - Definição dos Indicadores de Análise

\begin{tabular}{|c|c|c|c|}
\hline ÁREAS & TIPO & $\begin{array}{l}\text { INDICADORES } \\
\text { QUANTITATIVOS }\end{array}$ & $\begin{array}{l}\text { INDICADORES } \\
\text { QUALITATIVOS }\end{array}$ \\
\hline \multirow{25}{*}{ 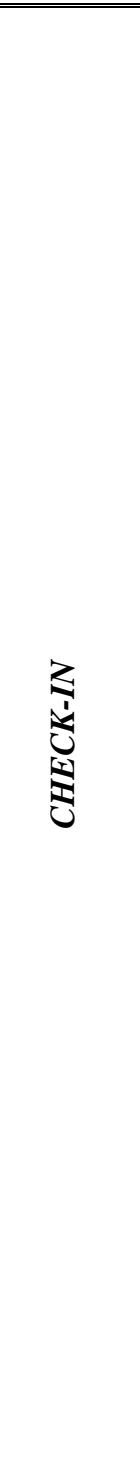 } & \multirow{13}{*}{ Tradicional } & \multirow{7}{*}{ Tempo de fila } & Tempo de espera na fila \\
\hline & & & Tempo de atendimento \\
\hline & & & Forma de organização da fila \\
\hline & & & Espaço disponível para a fila \\
\hline & & & Sinalização do balcão \\
\hline & & & Localização do balcão \\
\hline & & & Quantidade de balcões em operação \\
\hline & & \multirow{6}{*}{$\begin{array}{l}\text { Tempo de atendimento } \\
\text { (balcão) }\end{array}$} & Espaço disponível para o atendimento \\
\hline & & & Quantidade de funcionários em atendimento \\
\hline & & & Cordialidade e presteza no atendimento \\
\hline & & & Formas de priorização no atendimento \\
\hline & & & Clareza/precisão sobre as informações do voo \\
\hline & & & Aparência da área de check-in \\
\hline & \multirow{12}{*}{ Autoatendimento } & \multirow{3}{*}{ Tempo de fila (totem) } & Tempo de espera na fila \\
\hline & & & Forma de organização da fila \\
\hline & & & Tempo gasto no totem \\
\hline & & \multirow{3}{*}{$\begin{array}{l}\text { Tempo de atendimento } \\
\text { (totem) }\end{array}$} & Quantidade de totens em operação \\
\hline & & & Espaço disponível para o autotendimento \\
\hline & & & Sinalização indicativa da localização totens \\
\hline & & \multirow{3}{*}{$\begin{array}{l}\text { Tempo de fila (despacho } \\
\text { de bagagens) }\end{array}$} & Localização dos totens \\
\hline & & & Facilidade de manuseio do equipamento \\
\hline & & & Disponibilidade de funcionários para auxílio \\
\hline & & \multirow{3}{*}{$\begin{array}{l}\text { Tempo de atendimento } \\
\text { (despacho de bagagens) }\end{array}$} & Habilidade dos funcionários no auxílio \\
\hline & & & Confiabilidade do Serviço \\
\hline & & & Tempo de espera no despacho de bagagem \\
\hline \multirow{8}{*}{ 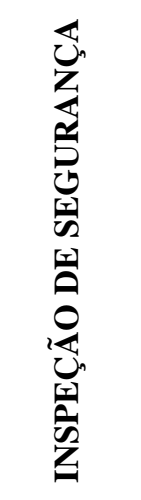 } & \multirow{8}{*}{-} & \multirow{4}{*}{ Tempo de fila } & Tempo de espera na fila \\
\hline & & & Forma de organização da fila de espera \\
\hline & & & Espaço disponível para a fila de espera \\
\hline & & & Tempo de atendimento \\
\hline & & \multirow{4}{*}{$\begin{array}{l}\text { Tempo de Atendimento } \\
\text { (raio-x) }\end{array}$} & Quantidade de funcionários em atendimento \\
\hline & & & Confiança nos procedimentos de vistoria \\
\hline & & & Quantidade de esteiras/pórticos em operação \\
\hline & & & $\begin{array}{l}\text { Cordialidade e presteza nos procedimentos de } \\
\text { vistoria }\end{array}$ \\
\hline
\end{tabular}




\section{Tabela 5 - Definição dos Indicadores da Área de Check-in Tradicional}

\begin{tabular}{|c|c|}
\hline $\begin{array}{c}\text { INDICADORES } \\
\text { QUANTITATIVOS } \\
\end{array}$ & DEFINIÇÃO \\
\hline Tempo de fila (balcão) & $\begin{array}{l}\text { É o tempo entre o momento que o passageiro pára na fila do } \\
\text { check-in até o momento em que é chamado para o } \\
\text { atendimento no balcão. }\end{array}$ \\
\hline Tempo de atendimento (balcão) & $\begin{array}{l}\text { É o tempo entre o momento em que o passageiro começa a } \\
\text { ser atendido no balcão até a emissão do seu bilhete aéreo e } \\
\text { pesagem das bagagens. }\end{array}$ \\
\hline $\begin{array}{l}\text { INDICADORES } \\
\text { QUALITATIVOS }\end{array}$ & DEFINIÇÃO \\
\hline Tempo de espera na fila & $\begin{array}{l}\text { É a percepção que o passageiro tem sobre o tempo em que } \\
\text { ficou na fila aguardando ser atendido pelo funcionário da } \\
\text { companhia área no balcão. }\end{array}$ \\
\hline Tempo de atendimento & $\begin{array}{l}\text { É a percepção que o passageiro tem sobre o tempo em que } \\
\text { ficou em atendimento no balcão. }\end{array}$ \\
\hline Forma de organização da fila & $\begin{array}{l}\text { É a percepção que o passageiro tem sobre a forma em que foi } \\
\text { organizada a fila do check-in. }\end{array}$ \\
\hline Espaço disponível para a fila & $\begin{array}{l}\text { É a percepção que o passageiro tem sobre o espaço } \\
\text { disponível para a fila do check-in. }\end{array}$ \\
\hline Sinalização do balcão & $\begin{array}{l}\text { É a percepção que o passageiro tem sobre a sinalização da } \\
\text { área de balcões do check-in, ou seja, se o balcão para o qual o } \\
\text { passageiro tem que se dirigir está bem sinalizado (sinalização } \\
\text { indicativa de balcão exclusivo para despacho de bagagens, } \\
\text { por exemplo). }\end{array}$ \\
\hline Localização do balcão & $\begin{array}{l}\text { É a percepção que o passageiro tem sobre a localização dos } \\
\text { balcões do check-in da companhia aérea, ou seja, este item } \\
\text { julga se o balcão está bem posicionado no terminal de } \\
\text { passageiros. }\end{array}$ \\
\hline Quantidade de balcões em operação & $\begin{array}{l}\text { Retrata a satisfação que o passageiro tem em relação ao } \\
\text { número de balcões que estavam em operação no momento } \\
\text { em que foi atendido. }\end{array}$ \\
\hline Espaço disponível para o atendimento & $\begin{array}{l}\text { É a percepção que o passageiro tem sobre o espaço } \\
\text { disponível para o atendimento no balcão. Trata-se do esaço } \\
\text { disponível entre o balcão e o limite da fila. }\end{array}$ \\
\hline Quantidade de funcionários em atendimento & $\begin{array}{l}\text { Retrata a satisfação que o passageiro tem em relação ao } \\
\text { número de atendentes que estão atuando no momento em que } \\
\text { foi atendido. }\end{array}$ \\
\hline Cordialidade e presteza no atendimento & $\begin{array}{l}\text { É a percepção que o passageiro tem sobre a cordialidade e } \\
\text { presteza do(s) funcionário(s) da companhia aérea. }\end{array}$ \\
\hline Formas de priorização no atendimento & $\begin{array}{l}\text { É a percepção que o passageiro tem sobre os tipos de } \\
\text { priorização que a companhia aérea tem para seus clientes no } \\
\text { atendimento do check-in. }\end{array}$ \\
\hline Clareza/precisão sobre as informações do voo & $\begin{array}{l}\text { É a percepção que o passageiro tem sobre a clareza ou a } \\
\text { precisão das informações relacionadas ao seu voo. }\end{array}$ \\
\hline Aparência da área de $c h e c k$-in & $\begin{array}{l}\text { É a percepção que o passageiro tem sobre a aparência geral } \\
\text { do check-in relacionadas à iluminação, limpeza, organização } \\
\text { geral e estética. }\end{array}$ \\
\hline
\end{tabular}




\section{Tabela 6 - Definição dos Indicadores da Área de Check-in de Autoatendimento}

\begin{tabular}{|c|c|}
\hline $\begin{array}{c}\text { INDICADORES } \\
\text { QUANTITATIVOS }\end{array}$ & DEFINIÇÃO \\
\hline Tempo de fila (totem) & $\begin{array}{l}\text { É o tempo entre o momento que o passageiro pára na fila do } \\
\text { check-in até o momento em que começa a operação de } \\
\text { atendimento no totem. }\end{array}$ \\
\hline Tempo de atendimento (totem) & $\begin{array}{l}\text { É o tempo entre o momento em que o passageiro começa a } \\
\text { utilizar o totem até a emissão do seu bilhete aéreo. }\end{array}$ \\
\hline Tempo de fila (despacho de bagagens) & $\begin{array}{l}\text { É o tempo entre o momento que o passageiro pára na fila do } \\
\text { para despachar a bagagem até o momento em que é atendido } \\
\text { no balcão. }\end{array}$ \\
\hline Tempo de atendimento (despacho de bagagens) & $\begin{array}{l}\text { É o tempo entre o momento em que o passageiro é atendido } \\
\text { no balcão até o despacho da bagagem. }\end{array}$ \\
\hline $\begin{array}{l}\text { INDICADORES } \\
\text { QUALITATIVOS }\end{array}$ & DEFINIÇÃO \\
\hline Tempo de espera na fila & $\begin{array}{l}\text { É a percepção que o passageiro tem sobre o tempo em que } \\
\text { ficou na fila aguardando a utilização da máquina de } \\
\text { autoatendimento (totem) da companhia área. }\end{array}$ \\
\hline Forma de organização da fila & $\begin{array}{l}\text { É a percepção que o passageiro tem sobre a forma em que foi } \\
\text { organizada a fila do check-in. }\end{array}$ \\
\hline Tempo gasto no totem & $\begin{array}{l}\text { É a percepção que o passageiro tem sobre o tempo em que } \\
\text { ficou utilizando a máquina de autoatendimento (totem) da } \\
\text { companhia área. }\end{array}$ \\
\hline Quantidade de totens em operação & $\begin{array}{l}\text { Retrata a satisfação do passageiro quanto ao número de totens } \\
\text { que estavam em operação no momento em que o passageiro } \\
\text { foi atendido. }\end{array}$ \\
\hline Espaço disponível para o autotendimento & $\begin{array}{l}\text { É a percepção que o passageiro tem sobre o espaço disponível } \\
\text { para a área de atendimento da companhia área. }\end{array}$ \\
\hline Sinalização indicativa da localização totens & $\begin{array}{l}\text { É a percepção que o passageiro tem sobre a sinalização } \\
\text { indicativa da área de autoatendimento da companhia área. }\end{array}$ \\
\hline Localização dos totens & $\begin{array}{l}\text { É a percepção que o passageiro tem sobre a localização dos } \\
\text { totens da companhia aérea. }\end{array}$ \\
\hline Facilidade de manuseio do equipamento & $\begin{array}{l}\text { É a percepção que o passageiro tem sobre a facilidade de } \\
\text { executar a máquina de autoatendimento da companhia aérea. }\end{array}$ \\
\hline Disponibilidade de funcionários para auxílio & $\begin{array}{l}\text { Retrata a satisfação do passageiro sobre a disponibilidade de } \\
\text { funcionários para auxiliá-lo no check-in de autoatendimento, } \\
\text { caso necessite. }\end{array}$ \\
\hline Habilidade dos funcionários no auxílio & $\begin{array}{l}\text { É a percepção que o passageiro tem sobre a habilidade dos } \\
\text { funcionários no auxílio do check-in de autoatendimento da } \\
\text { companhia aérea. }\end{array}$ \\
\hline Confiabilidade do Serviço & $\begin{array}{l}\text { É a percepção que o passageiro tem sobre a confiança no } \\
\text { sistema de autoatendimento disponibilizado pela companhia } \\
\text { aérea. }\end{array}$ \\
\hline Tempo de espera no despacho de bagagem & $\begin{array}{l}\text { É a percepção que o passageiro tem sobre o tempo em que } \\
\text { ficou aguardando na fila para o despacho de bagagens, caso } \\
\text { tivesse. }\end{array}$ \\
\hline
\end{tabular}




\section{Tabela 7 - Definição dos Indicadores da Área de Inspeção de Segurança}

\begin{tabular}{|c|c|}
\hline $\begin{array}{c}\text { INDICADORES } \\
\text { QUANTITATIVOS }\end{array}$ & DEFINIÇÃO \\
\hline Tempo de fila & $\begin{array}{l}\text { É o tempo entre o momento que o passageiro pára na fila da } \\
\text { inspeção de segurança até o momento em que é chamado para } \\
\text { o atendimento na área de raio-x. }\end{array}$ \\
\hline Tempo de atendimento (raio-x) & $\begin{array}{l}\text { É o tempo entre o momento em que o passageiro começa a ser } \\
\text { atendido na área de raio-x até o momento em que sai do } \\
\text { módulo de raio-x com seus pertences (bagagem de mão e/ou } \\
\text { outros objetos pessoais). }\end{array}$ \\
\hline $\begin{array}{l}\text { INDICADORES } \\
\text { QUALITATIVOS } \\
\end{array}$ & DEFINIÇÃO \\
\hline Tempo de espera na fila & $\begin{array}{l}\text { É a percepção que o passageiro tem sobre o tempo em que } \\
\text { ficou na fila da inspeção de segurança aguardando ser } \\
\text { atendido. }\end{array}$ \\
\hline Forma de organização da fila de espera & $\begin{array}{l}\text { É a percepção que o passageiro tem sobre a forma em que foi } \\
\text { organizada a fila da inspeção de segurança. }\end{array}$ \\
\hline Espaço disponível para a fila de espera & $\begin{array}{l}\text { É a percepção que o passageiro tem sobre o espaço disponível } \\
\text { para a fila da inspeção de segurança. }\end{array}$ \\
\hline Tempo de atendimento & $\begin{array}{l}\text { É a percepção que o passageiro tem sobre o tempo em que } \\
\text { ficou em atendimento no módulo de raio-x. }\end{array}$ \\
\hline Quantidade de funcionários em atendimento & $\begin{array}{l}\text { Retrata a satisfação do passageiro quanto ao número de } \\
\text { atendentes que estão atuando no momento em que foi } \\
\text { atendido. }\end{array}$ \\
\hline Confiança nos procedimentos de vistoria & $\begin{array}{l}\text { É a percepção que o passageiro tem sobre a confiança nos } \\
\text { procedimentos de vistoria da inspeção de segurança. }\end{array}$ \\
\hline $\begin{array}{l}\text { Cordialidade e presteza nos procedimentos de } \\
\text { vistoria }\end{array}$ & $\begin{array}{l}\text { É a percepção que o passageiro tem sobre a cordialidade e } \\
\text { presteza dos funcionários da área de inspeção de segurança. }\end{array}$ \\
\hline Quantidade de esteiras/pórticos em operação & $\begin{array}{l}\text { Retrata a satisfação do passageiro quanto ao número de } \\
\text { esteiras de bagagens de mão e de pórticos em funcionamento } \\
\text { no momento em que foi atendido. }\end{array}$ \\
\hline
\end{tabular}

\subsection{Método de coleta}

O método de coleta desta pesquisa foi composto por: registro das observações em campo, entrevista com passageiros, cronometragem do tempo de espera e de atendimento dos passageiros entrevistados e registro fotográfico das áreas de estudo no TPS. Tal pesquisa ocorreu de forma simultânea por meio de três equipes compostas por um coordenador e estagiários em cada um dos aeroportos. A coleta de dados foi realizada nos períodos matutino, diurno e noturno de 29 de setembro até o dia 04 de outubro de 2009. 
O questionário de pesquisa foi desenvolvido com base nos indicadores selecionados (vide Tabela 1). Os indicadores de cada área de estudo foram avaliados por meio de entrevistas com passageiros. Estes atribuíram uma nota para cada indicador, que variou de 1 a 5, que conceitualmente, equivale de ruim a excelente.

\subsection{Dimensionamento da amostra}

Para determinação do tamanho da amostra é utilizada a Equação (1), na qual fundamenta-se na estimativa da proporção populacional.

$$
n=\frac{N p q\left(Z_{\alpha / 2}\right)^{2}}{p q\left(Z_{\alpha / 2}\right)^{2}+(N-1) E^{2}}
$$

em que,

- n: tamanho da amostra;

- $\mathrm{Z}_{\alpha / 2}$ : valor padronizado correspondente a um determinado nível de confiança $\alpha$;

- E: erro da amostra;

- p e q: proporção da população com as características que estão sendo analisadas. No caso de uma proporção desconhecida, adota-se como hipótese: $\mathrm{p}=50 \%$ ou $\mathrm{p}$ $=0,5$; ("p" é complementar de "q");

- $\mathrm{N}=$ tamanho da população.

O número total de passageiros observados e entrevistados foi de 1.281 passageiros, sendo 694 em SBGR, 281 em SBSP e 306 em SBBR. O erro real de cada amostra, baseado no número total de passageiros que efetivamente embarcaram, segundo a ANAC (2009), nos referidos aeroportos durante o período, foram de: 3,8\% em SBGR, 5,9\% em SBSP e 5,7\% em SBBR.

\subsection{Método de análise: regressão linear}

Para esta pesquisa selecionou-se como método de análise a técnica de "Regressão Linear", que utiliza a relação entre duas ou mais variáveis quantitativas (ou qualitativas), de tal forma que uma variável pode ser predita a partir de outra(s). Esse método é representado pela Equação 2 a seguir. 
em que,

$$
\begin{aligned}
& Y_{i}=\beta_{0}+\beta_{1} X_{i}+\varepsilon_{i} \\
& \mathrm{i}=1,2, \ldots, \mathrm{n}
\end{aligned}
$$

i. Yi é o i-ésimo valor da variável resposta ou variável dependente;

ii. $\beta 0$ e $\beta 1$ são os parâmetros (coeficientes de regressão);

iii. Xi é o i-ésimo valor da variável preditora ou independente;

iv. $\varepsilon$ i é o termo do erro aleatório. A hipótese de autocorrelação do termo do erro aleatório considerada foi independente e normalmente distribuído, com hipótese da média zero do termo de erro igual a zero e variância constante.

A análise de regressão linear considerou como variáveis dependentes medidas qualitativas (opinião dos passageiros) de forma que foram testadas algumas relações entre as medidas qualitativas e quantitativas. Para a aplicação da regressão foi utilizada a técnica na qual, inicialmente, todas as variáveis eram incorporadas ao modelo. Nas etapas seguintes as variáveis eram eliminadas até o momento em que não existiam variáveis a serem excluídas. O processo era, então, interrompido e as variáveis restantes definiram o modelo final.

A seguir, são apresentados os modelos testados para o check-in tradicional, check-in autoatendimento e inspeção de segurança para o setor doméstico do Aeroporto Internacional de Brasília e do Aeroporto de São Paulo/Congonhas. Adicionalmente, foram adicionados modelos para o setor internacional do Aeroporto Internacional de São Paulo/Guarulhos.

\subsection{Modelos testados nos aeroportos de estudo}

Um total de três modelos foram testados, segundo observações nos check-in tradicional, check-in autoatendimento e na inspeção de segurança dos aeroportos. A Tabela 8 apresenta os modelos testados de acordo com a área de estudo. 


\section{Tabela 8. Modelos testados por Área de Estudo}

\begin{tabular}{l|l} 
Modelos & \multicolumn{1}{c}{ Áreas } \\
\hline Modelo 1 & $\begin{array}{l}\text { Check-in tradicional doméstico } \\
\text { Check-in tradicional internacional } \\
\text { Check-in autoatendimento } \\
\text { Inspeção de Segurança }\end{array}$ \\
\hline Modelo 2 & $\begin{array}{l}\text { Check-in tradicional doméstico } \\
\text { Check-in tradicional internacional } \\
\text { Check-in autoatendimento } \\
\text { Inspeção de Segurança }\end{array}$ \\
\hline Modelo 3 & Check-in autoatendimento
\end{tabular}

Os modelos 1 e 2 de regressão linear para o check-in tradicional têm como variáveis dependentes a percepção do tempo de espera na fila e a percepção do tempo de atendimento, respectivamente. Especialmente para os modelos 1 e 2 do check-in tradicional internacional as variáveis independentes descritas como "fila 1" e "fila 2" referem-se à fila de pré-check-in e à fila principal de check-in, respectivamente. E, consequentemente, as variáveis independentes descritas com "atendimento 1" e "atendimento 2" referem-se ao pré-atendimento e ao atendimento principal, respectivamente.

Os modelos 1, 2 e 3 para o check-in autoatendimento analisam as variáveis dependentes "percepção do tempo de espera na fila do totem", a percepção do tempo gasto no totem e a percepção do "tempo de espera na fila do despacho de bagagem".

Os modelos 1 e 2 de regressão linear para a área de inspeção de segurança têm como variáveis dependentes a "percepção do tempo de espera na fila de processamento" e a "percepção do tempo de atendimento", respectivamente. 


\section{i. Check-in tradicional doméstico - Modelo 1}

PTempo_esp CHECK-IN_DOM $=\beta_{0}+\beta_{1}$ Tempo_esp $+\beta_{2} \mathrm{Org}_{-} f+\beta_{3}$ Esp $-f+\beta_{4}$ PTempo_at + $\beta_{5}$ Tempo_at $+\beta_{6}$ Quant_b $+\beta_{7}$ Loc_b $+\beta_{8} \operatorname{Sin}_{-} b+\varepsilon_{i}$

em que,

- PTempo_esp: percepção do tempo de espera na fila;

- Tempo_esp: tempo de espera na fila;

- Org_f: forma de organização da fila;

- Esp_f: espaço disponível para a fila;

- PTempo_at: percepção do tempo de atendimento;

- Tempo_at: tempo de atendimento;

- Quant_b: quantidade de balcões em operação;

- Loc_b: localização do balcão; e,

- Sin_b: sinalização do balcão.

\section{ii. Check-in tradicional doméstico - Modelo 2}

PTempo_at CHECK-IN_DOM $_{-}=\beta_{0}+\beta_{1}$ Tempo_at $+\beta_{2}$ Esp_at $+\beta_{3}$ Quant $\_$func $+\beta_{4}$ Formas prior

$+\beta_{5}$ Cord_at $+\beta_{6}$ Loc_b $+\beta_{7}$ Sin_ $b+\beta_{8} Q u a n t_{-} b+\beta_{9}$ Ptempo_esp $+\varepsilon_{i}$

em que,

- PTempo_at: percepção do tempo de atendimento;

- Tempo_at: tempo de atendimento;

- Esp_at: espaço disponível para o atendimento;

- Quant_func: quantidade de funcionários em atendimento;

- Formas_prior: formas de priorização no atendimento;

- Cord_at: cordialidade e presteza no atendimento;

- Loc_b: localização do balcão;

- Sin_b: sinalização do balcão;

- Quant_b: quantidade de balcões em operação; e,

- PTempo_esp: percepção do tempo de espera na fila. 


\section{iii. Check-in tradicional internacional - Modelo 1}

PTempo_esp CHECK-IN_INTERNACIONAL $=\beta_{0}+\beta_{1}$ Tempo_esp_1 $\beta_{2}$ Tempo_esp_2 $+\beta_{3}$ Org $f f+\beta_{4} E s p_{-} f+\beta_{5} P T e m p o \_a t+$ $\beta_{6}$ Tempo_at_1+ $\beta_{6}$ Tempo_at_2 $+\beta_{7} Q u a n t \_b+\beta_{8} L o c_{-} b+\beta_{9} \operatorname{Sin} \_b+\varepsilon_{i}$

em que,

- PTempo_esp: percepção do tempo de espera na fila;

- Tempo_esp_1: tempo de espera na fila 1;

- Tempo_esp_2: tempo de espera na fila 2;

- Org_f: forma de organização da fila;

- Esp_f: espaço disponível para a fila;

- PTempo_at: percepção do tempo de atendimento;

- Tempo_at_1: tempo de atendimento 1;

- Tempo_at_2: tempo de atendimento 2;

- Quant_b: quantidade de balcões em operação;

- Loc_b: localização do balcão; e,

- Sin_b: sinalização do balcão.

\section{iv. Check-in tradicional internacional - Modelo 2}

PTempo_at $t_{\text {CHECK-IN_INTERACIONAL }}=\beta_{0}+\beta_{1}$ Tempo_esp_1 $+\beta_{2}$ Tempo_esp_2 $+\beta_{3}$ Cord _at $+\beta_{4} E s p \_a t+\beta_{5} P T e m p o \_e s p+$ $\beta_{6}$ Tempo_at_1 $\beta_{6}$ Tempo_at_2 $+\beta_{7}$ Quant_b $+\beta_{8}$ Quant_func $+\beta_{9} L o c \_b+\beta_{10} \operatorname{Sin} \_b++\beta_{11}$ Formas_prior $+\varepsilon_{i}$

em que,

- PTempo_at: percepção do tempo de atendimento;

- Tempo_esp_1: tempo de espera na fila 1;

- Tempo_esp_2: tempo de espera na fila 2;

- Formas_prior: formas de priorização no atendimento;

- Esp_at: espaço disponível para o atendimento;

- PTempo_esp: percepção do tempo de espera na fila;

- Tempo_at_1: tempo de atendimento 1;

- Tempo_at_2: tempo de atendimento 2;

- Quant_b: quantidade de balcões em operação;

- Quant_func: quantidade de funcionários em atendimento

- Cord_at: cordialidade e presteza no atendimento; 
- Loc_b: localização do balcão; e,

- Sin_b: sinalização do balcão.

\section{v. Check-in autoatendimento - Modelo 1}

PTempo_esp $p_{\text {CHECK }-I N_{-} \text {AUTO }}=\beta_{0}+\beta_{1}$ Tempo $\_f+\beta_{2}$ PTempo $\_g t+\beta_{3}$ Tempo_at $+\beta_{4}$ Org $\_f+\beta_{5} Q u a n t \_t+\varepsilon_{i}$ em que,

- PTempo_esp: percepção do tempo de espera na fila;

- Tempo_f: tempo de espera na fila;

- PTempo_gt: percepção do tempo gasto no totem;

- Tempo_at: tempo de atendimento;

- Org_f: forma de organização da fila; e,

- Quant_t: quantidade de totens em operação.

\section{vi. Check-in autoatendimento - Modelo 2}

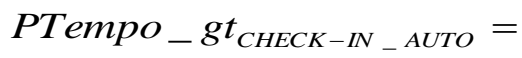
$\beta_{0}+\beta_{1}$ Tempo_at $+\beta_{2}$ Esp_at $+\beta_{3}$ Facil_man $+\beta_{4}$ Disp_func $+\beta_{5} H_{a} b_{-}$func $+\varepsilon_{i}$ em que,

- PTempo_gt: percepção do tempo gasto no totem;

- $\quad$ esp_at: espaço disponível para o autoatendimento;

- Hab_func: habilidade dos funcionários no auxílio;

- Facil_man: facilidade de manuseio do equipamento;

- Tempo_at: tempo de autoatendimento; e,

- Disp_func: disponibilidade de funcionários para auxílio.

\section{vii. Check-in autoatendimento - Modelo 3}

$$
\text { PTempo_espbag }_{\text {CHEC-IN_AUTO }}=\beta_{0}+\beta_{1} \text { Tempo_fbag }+\beta_{2} \text { Tempo_atbag }+\varepsilon_{i}
$$
em que,

- PTempo_espbag: percepção do tempo de espera no despacho de bagagem;

- Tempo_fbag: tempo de fila no despacho de bagagem; e,

- Tempo_atbag: tempo de atendimento no despacho de bagagem. 


\section{viii. Inspeção de Segurança - Modelo 1}

PTempo_esp $R_{R X \_D O M E S T I C O}=$

$\beta_{0}+\beta_{1}$ Tempo $_{-} f+\beta_{2} \mathrm{Org}_{-} f+\beta_{3}$ Esp $\_f+\beta_{4}$ PTempo_at $+\beta_{5}$ Tempo_at $+\beta_{6} Q u a n t_{-} m+\varepsilon_{i}$

em que,

- PTempo_esp: percepção do tempo de espera na fila;

- Tempo_f: tempo de espera na fila;

- Org_f: forma de organização da fila;

- Esp_f: espaço disponível para a fila;

- PTempo_at: percepção do tempo de atendimento;

- Tempo_at: tempo de atendimento; e,

- Quant_m: quantidade de módulos - esteiras e pórticos.

\section{ix. Inspeção de Segurança - Modelo 2}

PTempo_at $t_{\text {RX_DOMESTICO }}=\beta_{0}+\beta_{1}$ Tempo_at $+\beta_{2}$ Quant $_{-}$func $+\beta_{3}$ Cor_at $+\beta_{4}$ Quant_ $m+\beta_{5}$ Ptempo $-f+\varepsilon_{i}$ em que,

- PTempo_at: percepção do tempo de atendimento;

- Tempo_at: tempo de atendimento;

- Quant_func: quantidade de funcionários em atendimento;

- Cord_at: cordialidade e presteza no atendimento;

- Quant_m: quantidade de módulos - pórticos e esteiras em operação; e,

- PTempo_f: percepção de tempo de espera na fila.

\section{Análise de resultados}

Os resultados encontrados para a análise de regressão linear sobre as variáveis qualitativas e quantitativas dos aeroportos de estudo estão descritos na Tabela 9 para o Aeroporto Internacional de São Paulo/Guarulhos, na Tabela 10 para o Aeroporto Internacional de Brasília e na Tabela 11 para o Aeroporto de São Paulo/Congonhas. Os resultados apresentam as equações finais, a validação e as variáveis em ordem de importância dos componentes check-in tradicional, check-in autoatendimento e inspeção de segurança. 


\subsection{Aeroporto Internacional de São Paulo/Guarulhos}

Os fatores que afetam a percepção dos passageiros estão descritos nas equações dos modelos apresentados na Tabela 9. Os resultados indicam para o check-in tradicional doméstico o "tempo de fila" como indicador mais importante para a "percepção do tempo de fila" pelos passageiros no Modelo 1; e, a "percepção do tempo de fila" como indicador mais importante na avaliação da "percepção do tempo de atendimento" no Modelo 2. Para o check-in tradicional internacional a "percepção do tempo de atendimento" foi o indicador mais importante para a avaliação da "percepção do tempo de fila" no Modelo 1; e, a "cordialidade e presteza no atendimento" foi o indicador mais importante para a avaliação da "percepção do tempo de fila" no Modelo 2.

No check-in autoatendimento observou-se que o indicador "tempo de espera na fila" no Modelo 1 foi o mais importante na avaliação da "percepção do tempo de fila" pelos passageiros; assim como o indicador "habilidade do funcionário" no Modelo 2 foi para a "percepção do tempo gasto no totem" e o indicador "tempo de fila no despacho de bagagem" no Modelo 3 foi para “percepção do tempo de espera no despacho de bagagem”.

Na inspeção de segurança doméstico, observou-se que o indicador "organização da fila" foi o mais importante na avaliação da "percepção do tempo de fila" pelos passageiros no Modelo 1, assim como o indicador "percepção do tempo de fila" no Modelo 2 foi o mais importante na avaliação da "percepção do tempo de atendimento". Na análise da área de inspeção de segurança internacional observou-se no Modelo 1 que a "percepção do tempo de atendimento" foi o indicador mais importante para a avaliação da "percepção do tempo de fila" pelos passageiros, assim como o indicador "percepção do tempo de fila" foi para a “percepção do tempo de atendimento" no Modelo 2. 
Tabela 9. Resultados da Análise de Regressão Linear para o Aeroporto Internacional de São Paulo/Guarulhos

\begin{tabular}{|c|c|c|}
\hline & Componente & Equação \\
\hline \multirow{11}{*}{ 举 } & \multirow{2}{*}{$\begin{array}{l}\text { check-in } \\
\text { tradicional } \\
\text { doméstico }\end{array}$} & Modelo 1: PTempo_esp $=0,177+0,80 * P T e m p o \_a t+0,11 *$ Tempo_at $-1,30 *$ Tempo_esp \\
\hline & & Modelo 2: PTempo_at $=1,73+0,42$ Ptempo_esp $+0,26 * L o c_{-} b-0,11$ Tempo_at \\
\hline & \multirow{2}{*}{$\begin{array}{c}\text { check-in } \\
\text { tradicional } \\
\text { internacional }\end{array}$} & $\begin{array}{l}\text { Modelo 1: PTempo_esp }=0,727+0,476 \text { PTempo_at }+0,035 T e m p o_{-} a t_{-} 2+ \\
+0,33 \text { Org }{ }_{-} f-0,075 T e m p o_{-} e s p_{-} 1-0,039 T e m p o_{-} e s p_{-} 2\end{array}$ \\
\hline & & $\begin{array}{l}\text { Modelo 2: PTempo_esp }=1,59+0,376 \text { Cord } o_{-} a t+0,315 E s p_{-} a t- \\
\text {-0,048Tempo_esp_2-0,05Tempo_at_2-0,043Tempo_at_1 }\end{array}$ \\
\hline & & Modelo 1: PTempo_esp $=1,129+0,700 r g_{-} f-0,086$ Tempo_f \\
\hline & & Modelo 2: PTempo_esp $p_{C H E C K-I N \_A U T O}=1,696+0,52 E s p_{-} a t+0,168 H a b_{-}$func \\
\hline & & Modelo 3: PTempo_esp $=1,696-0,127$ Tempo_fbag -0,065Tempoatbag \\
\hline & \multirow{2}{*}{$\begin{array}{l}\text { inspeção de } \\
\text { segurança } \\
\text { doméstico }\end{array}$} & $\begin{array}{l}\text { Modelo 1: } \\
\text { PTempo_esp }=0,059-0,132 * T_{e m p o} f+0,570 * \text { Org }_{-} f+0,404 * P T e m p o \_a t\end{array}$ \\
\hline & & Modelo 2: PTempo_at $=0,863+0,450 *$ Quant $_{-}$func $+0,456 *$ Cor_at \\
\hline & \multirow{2}{*}{$\begin{array}{l}\text { inspeção de } \\
\text { segurança } \\
\text { internacional }\end{array}$} & 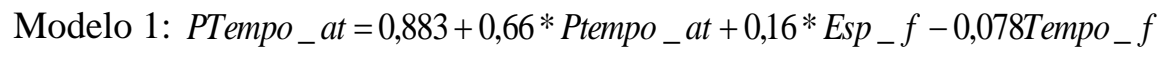 \\
\hline & & $\begin{array}{l}\text { Modelo 2: } \\
\text { PTempo_at }=0,592+0,659 * \text { Ptempo }_{-} f+0,208 * \text { Cord_at }-0,114 \text { Tempo_at }\end{array}$ \\
\hline
\end{tabular}

\subsection{Aeroporto Internacional de Brasília}

No check-in tradicional o indicador mais importante para a avaliação da "percepção do tempo de fila" no Modelo 1 foi a "forma de organização da fila", e no Modelo 2 o indicador mais importante foi "percepção do tempo de espera na fila" para a avaliação da percepção do tempo de atendimento.

No check-in autoatendimento o Modelo 1 foi excluído pois não houve fila significativa de passageiros durante o processamento nos totens, de forma que não foi possível realizar a avaliação da variável dependente "percepção do tempo de espera na fila do totem” para este aeroporto. Para os modelos 2 e 3, apenas uma variável dependente fez parte de cada modelo final, sendo respectivamente "localização dos totens" e "tempo de espera no despacho de bagagem".

Na área de inspeção de segurança o Modelo 1, em que a "percepção do tempo de espera na fila" é a variável dependente, foram determinadas como variáveis independentes a forma de organização da fila e o tempo de atendimento. Para o Modelo 2, as variáveis independentes determinadas foram "percepção do tempo de espera na fila" e "quantidade de funcionários em 
atendimento", em ordem de importância, as quais foram consideradas diretamente relacionadas a variável dependente "percepção do tempo de atendimento".

\section{Tabela 10. Resultados da Análise de Regressão Linear para o Aeroporto Internacional de Brasília}

\begin{tabular}{|c|c|c|}
\hline & Componente & Equação \\
\hline \multirow{6}{*}{ 哭 } & \multirow{2}{*}{$\begin{array}{l}\text { check-in } \\
\text { tradicional } \\
\text { doméstico }\end{array}$} & Modelo 1: PTempo_f $f=0,95+0,46 *$ org $\_f+0,34 * l o c_{-} b-0,027 *$ tempo_f \\
\hline & & Modelo 2: $P$ Tempo_at $=2,46+0,50 *$ Ptempo $_{-} f-0,06 *$ Tempo_at \\
\hline & \multirow{2}{*}{$\begin{array}{c}\text { check-in } \\
\text { autoatendimento }\end{array}$} & Modelo 2: PTempo_gt $=3,30+0,31 * l o c_{-} t$ \\
\hline & & Modelo 3: PTempo_espbag $=3,11+0,04 * t e m p o \_f b a g$ \\
\hline & \multirow{2}{*}{$\begin{array}{l}\text { inspeção de } \\
\text { segurança }\end{array}$} & Modelo 1: PTempo $_{-} f=0,839+0,679 *$ org $_{-} f+0,163 *$ ptempo_at \\
\hline & & Modelo 2: PTempo_at $=0,237+0,627 *$ ptempo_ $f+0,267 *$ quant_func \\
\hline
\end{tabular}

\subsection{Aeroporto de São Paulo/Congonhas}

Quanto aos resultados da análise de regressão para o check-in tradicional, verifica-se que os fatores que afetam na percepção do passageiro quanto ao Modelo 1 por ordem de importância são: (1) a percepção do passageiro quanto ao tempo de atendimento, (2) a organização da fila e (3) o tempo que ele realmente permaneceu em fila. Para o Modelo 2, os indicadores "cordialidade e presteza no atendimento" e o "espaço disponível para fila" são aqueles de maior importância para o passageiro, ao considerar o tempo de atendimento do check-in tradicional.

Para o check-in autoatendimento, o Modelo 1 constatou que os fatores que afetam a percepção do passageiro quanto ao tempo de espera na fila, por ordem de importância são: (1) a organização da fila e (2) o tempo de espera na fila. Para o Modelo 2, nota-se que os indicadores "espaço disponível para o atendimento" e "tempo de atendimento" são aqueles de maior importância para o passageiro, nessa ordem respectivamente, ao considerar o tempo gasto no check-in autoatendimento. Em relação ao Modelo 3, o resultado da análise de regressão, mostra que o "tempo de espera na fila de bagagem" é o indicador que representa maior importância na percepção do passageiro quanto ao tempo de espera.

No que diz respeito à inspeção de segurança, os fatores que afetam na percepção do passageiro quanto ao tempo de espera na fila por ordem de importância são: (1) a percepção 
do tempo de atendimento, (2) o tempo de permanência na fila de espera e (3) a organização da fila. Quanto ao Modelo 2, os indicadores "quantidade de funcionários no atendimento" e “cordialidade e presteza no atendimento" são aqueles, em ordem decrescente respectivamente, de maior importância para o passageiro, ao considerar o tempo de atendimento na área de inspeção de segurança.

Tabela 11. Resultados da Análise de Regressão Linear para o Aeroporto de São Paulo/Congonhas

\begin{tabular}{|c|c|c|}
\hline & Componente & Equação \\
\hline \multirow{7}{*}{ ज्ञ } & \multirow{2}{*}{$\begin{array}{l}\text { check-in } \\
\text { tradicional } \\
\text { doméstico }\end{array}$} & $\begin{array}{l}\text { Modelo 1: } \\
\text { PTempo_esp }=0,583-0,058 * \mathrm{Tempo}_{-} f+0,254 * \mathrm{Org}_{-} f+0,638 * \mathrm{PTempo}_{-} a t\end{array}$ \\
\hline & & $\begin{array}{l}\text { Modelo 2: } \\
\text { PTempo_at }=1,029+0,052 * \text { Tempo_at }+0,101 * \text { Org _ } f+0,275 * E s p_{-} f+ \\
+0,275 * \text { Cord_at }+0,138 * \text { PTempo_esp }^{-}\end{array}$ \\
\hline & \multirow{3}{*}{$\begin{array}{l}\text { check-in } \\
\text { autoatendimento }\end{array}$} & Modelo 1: PTempo_esp $=1,538-0,164 *$ Tempo _ $f+0,723 *$ Org $\_f$ \\
\hline & & Modelo 2: PTempo $_{-} g t=3,461-0,343 *$ Tempo_at $+0,373 * E s p_{-}$at \\
\hline & & $\begin{array}{l}\text { Modelo 3: } \\
\text { PTempo_espbag }=3,36-0,133 * \text { Tempo_fbag }+0,169 * \text { Tempo_atbag }\end{array}$ \\
\hline & \multirow{2}{*}{$\begin{array}{l}\text { inspeção de } \\
\text { segurança }\end{array}$} & $\begin{array}{l}\text { Modelo 1: } \\
\text { PTempo_esp }=0,196-0,532 * \text { Tempo_f } f+0,385 * \mathrm{Org}_{-} f+0,614 \text { PTempo_at }+\varepsilon_{i}\end{array}$ \\
\hline & & $\begin{array}{l}\text { Modelo 2: } \\
\text { PTempo_ at } \\
\text { RX }=0,665+0,662 * \text { Quant_func }+0,178 * \text { Cor_at }\end{array}$ \\
\hline
\end{tabular}

\section{Conclusão}

O estudo apresentou uma análise sobre a percepção de passageiros aeroportuários em duas das principais áreas de embarque - check-in e inspeção de segurança. O objetivo do trabalho foi obter a importância de indicadores, qualitativos ou quantitativos, que afetam a percepção da qualidade de serviço no Aeroporto Internacional de São Paulo/Guarulhos - Governador André Franco Montoro (SBGR), Aeroporto de São Paulo/Congonhas (SBSP) e Aeroporto Internacional de Brasília/Presidente Juscelino Kubitschek (SBBR). 
Indicadores qualitativos e quantitativos, coletados durante a pesquisa de campo com passageiros, foram avaliados por meio do método de regressão linear. Desta modo, obteve-se resultados acerca das percepções e perspectivas dos passageiros, onde levou-se em consideração as características de cada aeroporto. É importante ressaltar que a pesquisa colaborou com o avanço em estudos voltados para o nível de serviço, no que diz respeito aos indicadores selecionados para o estudo de campo. Tais indicadores não tinham sido relatados pela literatura anteriormente.

Os modelos de regressão linear, propostos neste trabalho, indicaram que há uma relação forte entre a percepção, ou seja, a visão que o passageiro tem sobre o componente e/ou serviço oferecido no terminal de passageiros e o tempo decorrido, seja este de espera na fila ou de atendimento. Os resultados mostraram ainda, que cada aeroporto apresentou variáveis independentes diferentes atuando fortemente sobre as mesmas variáveis dependentes dos modelos analisadas.

Dentre as limitações do trabalho, destacam-se a heterocedasticidade não controlada, onde a não adequação dos modelos apresentados podem gerar parâmetros menos eficientes podendo ser detectado por meio de teste residual; e, a falta de clareza por parte dos passageiros quando avaliaram indicadores como "sinalização" e "localização", por exemplo. Observou-se forte correlação entre as mesmas durante a análise de correlação das variáveis. No entanto, eliminaram-se as variáveis do modelo em que estiveram fortemente correlacionadas evitando, assim, a multicolinearidade.

Por último, os resultados apresentados são úteis para avaliação da administração aeroportuária e são recomendados, nesta pesquisa, como fonte de planejamento para disponibilizar áreas, bem como para a otimização dos procedimentos operacionais desses aeroportos. Desta forma, seria possível garantir e oferecer aos usuários melhor qualidade de serviço, tornando-os mais adequados com a realidade de cada tipo de terminal aeroportuário.

\section{Agradecimentos}

Os autores agradecem aos funcionários da Infraero pelo apoio dado durante a pesquisa de campo nos aeroportos relacionados ao estudo deste trabalho. 


\section{Referências}

Agência Nacional de Aviação Civil - ANAC (2009) Movimento operacional dos principais aeroportos do Brasil. Relatório Anual (2005-2007). Disponível em < www.anac.gov.br>.

Airports Council International - ACI (2000) Quality of service at airports: standards \& measurements. ACI World Headquarters, Geneva, Switzerland.

Alves, C. J. P. (1981) Uma metodologia para dimensionamento e avaliação de terminais de passageiros em aeroportos brasileiros. Tese de Mestrado em Engenharia Civil, EPUSP, São Paulo.

Alves, C. J. P. e Fraga, R. (2012) Capacidade do lado aéreo dos aeroportos brasileiros. Journal of Transport Literature, vol. 6, n. 4, pp. 178-189.

Bandeira, M. C. G. da S. P. (2008) Análise do nível de serviço em terminais de passageiros aeroportuários. Dissertação de Mestrado. Instituto Tecnológico de Aeronáutica, São José dos Campos, 134f. São José dos Campos.

Bandeira, M. C. G. da S. P., Duarte, M. C., Correia, A. R. e Alves, C. J. P. (2008) Desenvolvimento de uma medida de nível de serviço para o terminal de passageiros do Aeroporto Internacional de Fortaleza. In: Congresso de Pesquisa e Ensino em Transportes, Fortaleza. Anais da XXII ANPET. vol. 1. pp. 1-2.

Bandeira, M., Correia, A. R. e Wirasinghe, S. C. (2007) Degree of importance of airport passenger terminal components and their attributes. In: 11th Annual World Conference - Air Transport Research Society (ATRS), Berkeley, CA. June.

Carvalho, B. G. (2006) Uma metodologia para obtenção de um diagnóstico dos principais aeroportos no Brasil através da avaliação da relação demanda e capacidade. Tese de Mestrado. Instituto Tecnológico de Aeronáutica, São José dos Campos.

Correia, A. R. (2005) Evaluation of level of service at airport passenger terminals: individual components and overall perspectives. Thesis (PhD) - University of Calgary, Canada.

Correia, A. R., Wirasinghe, S. C. e de Barros, A. G. (2006) A global index for level of service evaluation at airport passenger terminals. In: Proceedings of the $10^{\text {th }}$ ATRS Annual World Conference. Nagoya: ATRS, pp. 1-15. Nagoya.

Correia, A. R., Wirasinghe, S. C. e de Barros, A. G. (2007) A global index for level of service evaluation at airport passenger terminals. Transportation Research Part E, Logistics and Transportation Review, vol. 44, pp. 607-620.

Cravo, B. M. (2014) A alocação de slots e a concorrência no setor de transporte aéreo. Journal of Transport Literature, vol. 8, n. 1, pp. 159-177.

De Barros, A. G. e Tomber, D. D. (2007) Quantitative analysis of passenger and baggage security screening at airports. Journal of Advanced Transportation, Vol. 41, No. 2, 23 pp.

De Neufville, R. e Odoni, A. R. (2003) Airport systems: planning design and management. [S.1.]: McGraw-Hill Book.

Eller, R. A. G. e Moreira, M. (2014) The main cost-related factors in airlines management. Journal of Transport Literature, vol. 8, n. 1, pp. 8-23.

Empresa Brasileira de Infraestrutura Aeroportuária - INFRAERO (1988) Dimensionamento básico com o momento de maior solicitação. Brasília. 
Federal Aviation Administration - FAA (1988) Planning and design guidelines for airport terminal facilities. Advisory Circular.

Horonjeff, R. e McKelvey, F. X. (1994) Planning and design of airports. 4.ed. [S.1.]: McGraw-Hill.

International Air Transport Association - IATA (1995) Airport development reference manual. International Air Transport Association. 8. ed. Montreal, Geneva, April.

International Civil Airports Association - ICAA (1979) Methodology for determining the capacity of an airport. First working session presented by Mrs. Barbara Bodien, 19th ICAA Annual Congress, Nice.

Lee, S. C. e Kim, B. J. (2003) Development of passenger service quality index for Inchon International Airport. In: Annual Meeting. Proceedings of $82^{\text {nd }}$ Annual Meeting of the Transportation Research Board, TRB. Washington D. C., USA.

Lipovich, G. (2012) La calidad de los hubs de América Latina. Journal of Transport Literature, vol. 6, n. 2, pp. 144-168.

Linhares, G. C. (2012) Planejamento aeroportuário no Brasil: discussão sobre contribuições da prospectiva estratégica. Journal of Transport Literature, vol. 6, n. 4, pp. 147-163.

Magri Junior, A. A. (2003) Indicadores de qualidade de terminais de passageiros de aeroportos. Dissertação de Mestrado - Instituto Tecnológico de Aeronáutica, São José dos Campos.

Magri Junior, A. A. (2008) Impactos da aplicação do sistema check-in de autoatendimento em terminais de passageiros de aeroportos. Tese de doutorado. Universidade de São Paulo, USP, São Paulo.

Medeiros, A. G. M. (2004) Um método para dimensionamento de terminais de passageiros em aeroportos brasileiros. Dissertação de Mestrado - Instituto Tecnológico de Aeronáutica, São José dos Campos.

Medeiros, A. G. M. (2004) Um método para dimensionamento de terminais de passageiros em aeroportos brasileiros. Tese de Mestrado. Instituto Tecnológico de Aeronáutica, São José dos Campos.

Mumayiz, S. A. e Ashford, N. J. (1986) Methodology for planning and operations management of airport terminal facilities. Transportation Research Record 1094, TRB, National Research Council, Washington D. C., pp. 24-35.

Murça, M. C. R. e Correia, A. R. (2013) Análise da modelagem da escolha aeroportuária em regiões de múltiplos aeroportos. Journal of Transport Literature, vol. 7, n. 4, pp. 130-146.

Nascimento, M. V. (2013) Análise da competitividade do transporte aéreo com base no nível de serviço percebido pelo usuário. Journal of Transport Literature, vol. 7, n. 1, pp. 202-211.

Ndoh, N. N. e Ashford, N. J. (1994) Evaluation of transportation level of service using fuzzy sets. Transportation Research Record 1461, TRB, National Research Council, Washington, D.C., pp. 31-37.

Oliveira, A. V. M. e Turolla, F. A. (2013) Financiamento da infraestrutura de transportes. Journal of Transport Literature, vol. 7, n. 1, pp. 103-126.

Omer, K. F. e Khan, A. M. (1988) Airport landside level of service estimation: utility theoretic approach. Transportation Research Record 1199, TRB, National Research Council, Washington D. C., pp. 33-40.

Ramis, J. E. e Santos, E. A. (2013) The impact of thermal comfort in the perceived level of service and energy costs of three Brazilian airports. Journal of Transport Literature, vol. 7, n. 2, pp. 192-206. 
Ronzani, G. M. e Correia, A. R. (2007) Evaluating orientation level of service at passenger terminals at major Brazilian airports. Journal of the Brazilian Air Transportation Research Society, vol. 3, pp. 19-34.

Seneviratne, P. N. e Martel, N. (1991) Variables influencing performance of air terminal buildings. Transportation Planning and Technology, vol. 16, n. 1, pp. 3-28.

Spoljaric, E. A. (1998) Qualidade dos serviços nos terminais de passageiros de aeroportos. Tese de Mestrado - Instituto Tecnológico de Aeronáutica, São José dos Campos.

Transport Canada (1979) A discussion paper on level of service definition and methodology for calculating airport capacity. Report TP 2027.

Transportation Research Board - TRB (1987) Measuring airport landside capacity. Transportation Research Board - National Research Council, Special Report 215, Washington, D. C.

Wells, A. T. (1996) Airport planning and management, 3rd edition. London: McGraw-Hill.

Widmer, J. A. e Silva, I. (1989) An operational analysis of regional airport passenger terminal. USP - São Carlos.

Yeh, C. H. e Kuo, Y. L. (2003) Evaluation passenger services of Asia-Pacific international airports. Transportation Research Part E, vol. 39, pp. 35-48.

Yen, J. R., Teng, C. R. e Chen, P. S. (2001) Measuring the level of service at airport passenger terminals: comparison of perceived and observed time. National Research Council, Transportation Research Record n. 1744, Washington D.C., pp. 17-23. 